\title{
IP-receptor and PPARs trigger the conversion of human white to brite adipocyte induced by carbaprostacyclin
}

Rayane A. Ghandour ${ }^{1,2,3}$, Maude Giroud ${ }^{1,2,3}$, Alexandros Vegiopoulos ${ }^{4}$, Stephan Herzig $^{5,6,7,8}$, Gérard Ailhaud ${ }^{1,2,3}$, Ez-Zoubir Amri ${ }^{1,2,3^{*}}$ and Didier F. Pisani ${ }^{1,2,3^{*}}$

${ }^{1}$ Univ. Nice Sophia Antipolis, iBV, UMR 7277, 06100 Nice, France.

${ }^{2}$ CNRS, iBV UMR 7277, 06100 Nice, France.

${ }^{3}$ Inserm, iBV, U1091, 06100 Nice, France.

${ }^{4}$ DKFZ Junior Group Metabolism and Stem Cell Plasticity, German Cancer Research Center, Heidelberg, Germany,

${ }^{5}$ Institute for Diabetes and Cancer (IDC), Helmholtz Zentrum München, German Research Center for Environmental Health, Neuherberg, Germany

${ }^{6}$ Joint Heidelberg-IDC Translational Diabetes Program, Heidelberg University Hospital, Heidelberg, Germany

${ }^{7}$ Molecular Metabolic Control, Medical Faculty, Technical University Munich, Germany

${ }^{8}$ German Center for Diabetes Research (DZD), Neuherberg, Germany

"Corresponding authors: Dr. Didier Pisani, iBV; Université de Nice Sophia-Antipolis, UMR7277 CNRS - UMR1091 INSERM; Faculté de Médecine; 28 Avenue de Valombrose 06107 Nice cedex 2, France; Tel: +33 4933777 07; E-mail: pisani@unice.fr and Dr. Ez-Zoubir Amri, Tel: +33 49337 70 82; e-mail: amri@unice.fr.

Author contributions. The author(s) have made the following declarations about their contributions: Conceived and designed the experiments: DP, EZA. Performed the experiments: RAG, MG, AV, DFP. Analyzed the data: RAG, AV, SH, GA, DFP, EZA. Wrote the manuscript: RAG, GA, DFP, EZA.

Acknowledgements. This work was supported by CNRS, EU FP7 project DIABAT (HEALTH-F2-2011-278373) and Nutricia Research Foundation ("2011-25"). RAG is a recipient of a fellowship from the Association of Specialization and Scientific Orientation (Lebanon) and Ghazieh township (Lebanon). 


\begin{abstract}
.
Brite adipocytes recently discovered in humans are of considerable importance in energy expenditure by converting energy excess into heat. This property could be useful in the treatment of obesity, and nutritional aspects are relevant to this important issue. Using hMADS cells as a human cell model which undergoes a white to a brite adipocyte conversion, we had shown previously that arachidonic acid, the major metabolite of the essential nutrient $\omega 6$-linoleic acid, plays a major role in this process. Its metabolites PGE2 and PGF2 alpha inhibit this process via a calcium-dependent pathway, whereas in contrast carbaprostacyclin (cPGI2), a stable analog of prostacyclin, activates white to brite adipocyte conversion. Herein, we show that cPGI2 generates via its cognate cellsurface receptor IP-R, a cyclic AMP-signaling pathway involving PKA activity which in turn induces the expression of UCP1. In addition, CPGI2 activates the pathway of nuclear receptors of the PPAR family, i.e. PPARa and PPARy, which act separately from IP-R to up-regulate the expression of key genes involved in the function of brite adipocytes. Thus dual pathways are playing in concert for the occurrence of a browning process of human white adipocytes. These results make prostacyclin analogs as a new class of interesting molecules to treat obesity and associated diseases.
\end{abstract}

Key words. Prostacyclin, cPGI2, cAMP, brown adipocyte, obesity, UCP1. 


\section{INTRODUCTION}

White adipose tissue (WAT) stores and releases energy as lipids while brown adipose tissue (BAT) burns fat to produce heat by uncoupling the mitochondrial electron transport chain activity from ATP synthesis [1]. This energy dissipation is due to the unique expression of the uncoupling protein 1 (UCP1) in brown adipocyte mitochondria $[2,3]$. In addition to the thermogenic brown adipocytes located in BAT, WAT contains inducible thermogenic fat cells - called "brite" ("brown-in-white") or "beige" adipocytes which are able to metabolize fat and carbohydrates via non-shivering thermogenesis [47]. In vivo brite adipocytes appear in response to cold exposure, to chronic adrenergic stress or to high-fat diets and stem either from progenitors but mainly by direct conversion of mature white adipocytes [6, 8, 9]. During the last decade, various laboratories have shown that healthy adult humans display islets of energy-dissipating thermogenic adipocytes of metabolic significance [10-14], which display the signature of either classical brown or brite adipocytes depending on the localization and the depth of the analysed tissue [15-18]. As brown and brite adipocytes represent important candidates for controlling body weight, investigations on the regulation of brite adipocyte recruitment and activation in human are in demand, particularly from a nutritional point of view since quantitative and qualitative issues of dietary lipids are relevant to increased body weight [19].

We have recently shown that a metabolite arising from the essential $\omega 6$ linoleic acid (LA), i.e. arachidonic acid (ARA), inhibits the conversion of white to brite adipocytes [20]. The effect of ARA is mediated via cyclooxygenase (COX) activities leading to increased synthesis and release of inhibitory prostaglandins PGE2 and PGF2 $\alpha$, and finally activation of an oscillatory calcium pathway [20]. Interestingly, in vivo, COX pathway has been shown to be crucial for the induction of brite adipocytes in 129Sv mice, a mouse strain resistant to develop obesity due to its high content of brown and brite adipocytes [21, 22]. We had also shown earlier in rodents that ARA is active through prostacyclin synthesis and release to stimulate adipogenesis of white preadipocytes [23]. Prostacyclin acts through the IP-receptor (IP-R)/prostacyclin system, triggers CAMP production and activates the pro-adipogenic protein kinase $A(P K A)$ 
pathway [24, 25]. Both ex vivo and in vivo exposure of mouse white adipose tissue to carbaprostacyclin (cPGI2), a stable analogue of prostacyclin, stimulates the formation of adipocytes within few day [23, 26]. Moreover, ARA and some of its metabolites have been described in vitro as activators/agonists of peroxisome proliferator activated receptors (PPARs) stimulating adipogenesis of white preadipocytes [27]. However, compared to rodents, the mechanism by which cPGI2 favors the browning process of human white adipocytes remains largely unknown.

In the work described herein, we have investigated this process using a well described human cell model, i.e. hMADS cells [28, 29]. We show that, unlike PGE2 and PGF2 [20], cPGI2 up-regulates UCP1 expression and leads to increased thermogenesis of hMADS adipocytes by enhancing proton leak and basal respiration. Our results show that the conversion of white to brite adipocytes implicates two signaling pathways activated respectively by the cell surface prostacyclin receptor IP-R and by nuclear receptors of the PPAR family, i.e. PPARa and PPARy. 


\section{MATERIAL AND METHODS}

\subsection{Reagents.}

Culture media and buffer solutions were purchased from Lonza Verviers (Verviers, Belgium), fetal bovine serum, insulin and trypsin from Invitrogen (CergyPontoise, France), hFGF2 from Peprotech (Neuilly sur Seine, France). cPGI2, CAY10441, GW9662, MRE269 and rosiglitazone were purchased from Cayman (BertinPharma, Montigny le bretonneux, France). All the other products, when not indicated, were from Sigma-Aldrich (Saint Quentin Fallavier, France).

\subsection{Cell culture.}

The establishment, characterization and culture protocols of hMADS cells have been described previously [28, 30]. In the experiments reported herein hMADS-3 cells were used, originally isolated from the prepubic fat pad of a 4-month-old male. Cells were used between passages 14 and 20. Briefly, confluent cells were submitted to differentiation medium (DMEM/Ham's F12 media containing $10 \mu \mathrm{g} / \mathrm{ml}$ transferrin, $10 \mathrm{nM}$ insulin, and $0.2 \mathrm{nM}$ triiodothyronine) supplemented with $1 \mu \mathrm{M}$ dexamethasone and 500 $\mu \mathrm{M}$ isobutyl-methylxanthine. Two days later, the medium was changed, dexamethasone and isobutyl-methylxanthine were omitted and $100 \mathrm{nM}$ rosiglitazone were added for the indicated periods. Cells were treated between days 2 and 9 with rosiglitazone to enable white adipocyte differentiation to take place. After 5 days in its absence brite adipocyte conversion was induced by adding rosiglitazone between days 14 and 17. All compounds tested herein were added at day 14. Medium was changed every other day and cells were used at the indicated days.

Cytochrome C oxidase and Glycerol-3-phosphate dehydrogenase (GPDH) activity assays as well as Oil Red $O$ staining were performed as described previously $[29,31]$.

\subsection{Transfection of siRNAs.}

Transfection experiments were performed using HiPerfect (QIAGEN, Courtaboeuf, France) at days 12 and 14 of differentiation. Cells were incubated with a 
mixture containing HiPerfect and siRNA (20 nM) in DMEM. Four hours later, it was supplemented with F12 medium containing $20 \mu \mathrm{g} / \mathrm{ml}$ transferrin, $20 \mathrm{nM}$ insulin, and 0.4 $\mathrm{nM}$ triiodothyronine. siRNA against human PPARa consisted in a pool of 3 targetspecific 19-25 nucleotides siRNAs designed to knock- down gene expression (Santa Cruz, Heidelberg, Germany).

\subsection{Isolation and analysis of RNA.}

These procedures follow MIQE standard recommendations and were conducted as described previously [29]. Primer sequences are listed in Supplementary Table 1. Quantitative PCR (qPCR) were performed using SYBR qPCR premix Ex Taqll from Takara (Ozyme, Montigny-le-Bretonneux, France) and assays were run on a StepOnePlus ABI real-time PCR machine (PerkinElmer Life and Analytical Sciences, Boston). The expression of selected genes was normalized to that of $36 \mathrm{~B} 4$ housekeeping gene and then quantified using the comparative- $\Delta \mathrm{Ct}$ method.

\subsection{Western blot analysis.}

Cells were lysed in TNET lysis buffer (25 mM Tris-Cl (pH 7.4), $100 \mathrm{mM} \mathrm{NaCl}, 1$ mM EDTA, 1\% Triton X-100, 0.5\% Nonidet P40, 1x protease inhibitor cocktail and 1X Phosphostop mix (Roche Diagnostics, Meylan, France)). Protein concentration was evaluated by BCA assay according to manufacturer's recommendations (PIERCE, Thermo Scientific, France). Proteins were blotted using SDS-PAGE basic protocol. Primary antibody incubation was performed overnight at $4^{\circ} \mathrm{C}$ (anti-UCP1, Calbiochem (Merk-Millipore, Molsheim, France) \#662045, dilution 1:750; anti- $\beta$-tubulin, Sigma \#T5201, dilution 1:2000; anti-perilipin, Acris Antibodies (Interchim, Montluçon, France) \#BP5015, dilution 1:5000; anti-CREB, CellSignaling (Ozyme) \#9197, dilution 1:500; and anti-phospho-CREB, CellSignaling \#9190, dilution 1:500). Primary antibodies were detected with HRP-conjugated anti-rabbit or anti-mouse immunoglobulins (Promega, Charbonnières-les-Bains, France) and goat anti-guinea pig immunoglobulins (SantaCruz). Detection was performed using Immobilon Western Chemiluminescent HRP Substrate (Merk-Millipore). OD band intensities were evaluated using PCBas Software. 


\subsection{Mitochondrial respiration analysis.}

Oxygen consumption rate (OCR) of hMADS adipocytes was determined at day 17 using an XF24 Extracellular Flux Analyzer (Seahorse Bioscience, Proteigene, Saint Marcel, France) and normalised by protein content (evaluated by BCA assay). Uncoupled and maximal OCR was determined using oligomycin $(1.2 \mu \mathrm{M})$ and FCCP (1 $\mu \mathrm{M})$, respectively. Rotenone and Antimycin $\mathrm{A}$ (1 $\mu \mathrm{M}$ each) were used to inhibit Complex I- and Complex III-dependent respiration, respectively. Parameters were measured for each individual well using the different value of OCR as previously described [32].

\subsection{Mitochondrial DNA quantification.}

DNA was extracted using a DNA extraction kit (Macherey-Nagel EURL, Hoerdt, France). $2 \mathrm{ng}$ of total DNA were used for QPCR analysis, and the mitochondrial DNA content was determined as the ratio of the DNA content of the NADH dehydrogenase subunit 1 gene ( $N d 1$, mitochondrial DNA) to that of lipoprotein lipase gene ( $L p l, a$ nuclear DNA).

\subsection{Analysis of $\mathrm{Ca++}$ flux.}

hMADS cells were seeded in 4 well plates and differentiated until day 14 into adipocytes. Cells were incubated for 20 min at $37^{\circ} \mathrm{C}$ with $5 \mu \mathrm{M}$ of the fluorescent Ca++ sensitive probe Quest Fluo-8 (Euromedex, Souffelweyersheim, France) in PBS supplemented with $2 \mathrm{mM} \mathrm{CaCl} 2$ and $0.05 \%$ BSA. Cells were finally washed in PBS containing $2 \mathrm{mM} \mathrm{CaCl} 2$ and installed on the stage of an inverted microscope maintained at $37^{\circ} \mathrm{C}$ and illuminated with a xenon lamp through a dichroic filter (excitation $490 \mathrm{~nm}$ and emission over $510 \mathrm{~nm}$ ). Digital images were recorded every $5 \mathrm{~s}$ with a low light level camera (Photonic Science, St Etienne de St Geoirs, France) and processed for grey level analysis through imaging workbench software (v2.1).

\subsection{Statistical analyses.}


Data are expressed as mean values \pm SEM and were analyzed using InStat software (GraphPad, La Jolla, CA, USA). Data were analyzed by one-way ANOVA followed by a Student-Newman-Keuls post-test, or Student's t-test to assess statistical differences between experimental groups. Differences were considered statistically significant with $\mathrm{p}<0.05$. 


\section{RESULTS}

\section{1. $\quad c P G / 2$ induces the conversion of white to brite adipocytes.}

We have previously shown that rosiglitazone, a PPARy specific agonist, was able to induce the conversion of hMADS white adipocytes into britelbeige adipocytes as shown by a strong expression of UCP1 [29]. In order to test whether CPGI2 was able to substitute for rosiglitazone, hMADS cells first differentiated into white adipocytes in the presence of rosiglitazone from day 3 to day 9 , as described in Materials and Methods section, and then treated or not with increasing concentrations of $\mathrm{CPG} 2$ between days 14 and 17. UCP1 mRNA expression was analyzed at day 17 as an indicator of the degree of white to brite adipocyte conversion. As shown in Fig. 1A, UCP1 mRNA expression was induced in CPGI2-treated cells compared to untreated cells in a dosedependent manner though at lower expression levels than those observed when rosiglitazone was present between days 14 and 17. CPGI2 at $1 \mu \mathrm{M}$ induced UCP1 expression, at both mRNA and protein levels (Fig. 1A-B), corresponding to $20-30 \%$ of the maximal effect obtained upon rosiglitazone treatment. This concentration was routinely used in subsequent experiments.

In order to exclude any adipogenic effect of CPGI2 that could be associated with UCP1 induction, we analyzed cellular and molecular markers associated with adipogenesis at day 17. As shown in Fig. 1C, cells treated or not with CPGI2 harboured similar morphological differentiation (assessed by Oil red $O$ staining and phase contrast microscopy). Furthermore, cPGI2 treatment did not affect glycerol-3-phosphate dehydrogenase (GPDH) activity (Fig.1D), Perilipin A and B protein levels (Fig. 1B) as well as mRNA expression of several adipogenic markers (Fig. 1E).

\section{2. $\quad c P G I 2$ induces a functional brite adipocyte phenotype.}

To further characterize the effect of CPGI2 on the white to brite adipocyte conversion, we analyzed the mRNA expression levels of several brite adipocyte and mitochondrial markers. In addition to UCP1, CPGI2-treated adipocytes expressed higher mRNA levels of classical brite/brown markers (CPT1M, CIDEA, FABP3 and CITED1) (Fig. 2A). By contrast, ELOVL3, PAT2 and PRDM16 mRNA expression, three other brite 
adipocyte markers, was not affected by cPGI2 (Fig. 2A). The brite adipocyte phenotype of the cells was confirmed by the expression of specific marker Tbx1 and by the fact that Zic1, a brown adipocyte specific marker, was barely detectable (Fig. 2C).

mRNA expression of mitochondrial markers was not changed by $\mathrm{cPGI} 2$ treatment as shown for the mitochondrial transcription factor TFAM, TOMM20 (an ubiquitous mitochondrial translocator), citrate synthase (CS), COX10 and cytochrome C (2 components of respiratory chain complex IV) (Fig. 2B). However, a significant increase in the cytochrome $\mathrm{C}$ oxidase activity was found in CPGI2-treated adipocytes (Fig. 2D), suggesting a higher mitochondrial respiratory chain activity. In order to further characterize the mitochondrial status of hMADS brite adipocytes induced by cPGI2, oxygen consumption analysis was performed. As expected, cells display higher basal and maximal mitochondrial respiration rates, in line with the increased cytochrome $C$ oxidase activity (Fig. 2E and F). Moreover, the uncoupled respiration was two-fold higher in cPGI2-treated compared to untreated adipocytes, demonstrating a functional UCP1 protein (Fig. 2F). The higher mitochondrial respiration can be related at least in part to increased mitochondrial adipocyte content, as mitochondrial DNA content was higher in cPGI2-induced brite compared to control adipocytes (Fig. 2G).

\section{3. cPGI2 acts partially through the IP receptor.}

IP-R is a cell surface receptor coupled to $G$ s or $G q$ proteins [25]. We first demonstrated that intracellular $\mathrm{Ca}^{++}$concentration is not altered in hMADS adipocytes upon cPGI2 treatment, differently to ionomycin treatment which induced a transient increase in intracellular $\mathrm{Ca}^{++}$concentration (Fig. 3A). Also, we aimed to know whether other pathways could play a role. As shown in Fig.3, PKA pathway was activated upon acute cPGI2 exposure. CREB protein, known to be a target of the cAMP/PKA signaling pathway, was phosphorylated within 5 minutes and the effect was maintained for at least 30 minutes (Fig. 3B). In the presence of CAY10441 $(5 \mu \mathrm{M})$, an antagonist of IP-R, phosphorylation of CREB was delayed and disappeared thereafter (Fig. 3B). To further characterize the involvement of IP-R in cPGI2-induced browning of hMADS adipocytes, MRE269 as a highly specific IP-R agonist was used instead of cPGI2. As expected, MRE269 at $1 \mu \mathrm{M}$ induced a time-dependent CREB phosphorylation with a maximal 
effect at 30 minutes (Fig. 3B). As shown in Fig. 3B, treatment of hMADS adipocytes between days 14 and 17 with MRE269 allowed an increased UCP1 mRNA expression. In addition, the IP-R antagonist CAY10441 significantly down-regulated UCP1 mRNA expression induced by cPGI2. In both cases, the expression of CPT1M and PLN1 mRNA remained unchanged (Fig. 3C and D).

Thus it appears that the effect of the IP-R-activated pathway was confined to UCP1 expression, but not to that of genes associated to adipogenesis, likely due to the occurrence of a CREB responsive element in the UCP1 promoter [1]. Owing to the fact that the agonist MRE269 displayed only approximately $20 \%$ of the maximal cPGI2 effect on UCP1 expression and that the inhibitory effect of the antagonist CAY10441 on CREB phosphorylation was partial, these data strongly suggest that pathway(s) distinct from the IP-R signaling should be involved in the induction of UCP1 expression. This point was further investigated.

\subsection{PPARY and PPARa are part of the $C P G 12$ signaling.}

cPGl2 treatment did not affect adipogenesis per se as the expression of adipogenic marker genes remained unaltered (Fig. 1E), excepted FABP4 and adiponectin (Fig.4A) known to be PPARy targets. To highlight a potential cPGI2dependent activation of PPARY, hMADS adipocytes were exposed, between days 14 and 17, to cPGI2 and GW9662 at 300 nM, a PPARy antagonist. As expected, inhibition of PPARy activity decreased mRNA expression of FABP4 and adiponectin due to cPGI2 treatment (Fig. 4A). Moreover, inclusion of the PPARy antagonist significantly inhibited UCP1 and CPT1M mRNA expression induced by $\mathrm{CPGI} 2$ without affecting overall adipogenesis as shown by PLN1 expression (Fig.4B, C and D respectively). Interestingly, when cells were treated with both CAY10441 and GW9662, an additive effect of these antagonists on UCP1 mRNA expression was observed, but not for CPT1M mRNA expression (Fig. 4 B and C).

To assess whether PPARa, another member of the PPARs family, was also implicated, hMADS cells were transfected with $20 \mathrm{nM}$ of siRNA control or siRNA against PPAR $\alpha$ at days 12 and 14. Using this strategy, PPAR $a$ mNA levels decreased by more than $70 \%$ at day 17 (Fig. 5A). This lower expression of PPARa did not lead to a 
decrease in UCP1 mRNA (Fig. 5B) levels induced by cPGI2 treatment neither in PLN1 mRNA levels (Fig.5C), in contrast to the decrease observed for CPT1M mRNA expression (Fig. 5D). Moreover, co-inhibition of PPARy (using GW9662) activity and PPARa expression (using siRNA) led to a stronger inhibition of CPT1M mRNA expression suggesting an additive role of both PPARs in cPGI2-mediated effects (Fig. $5 D)$. 


\section{DISCUSSION}

Prostacyclin is known as a major metabolite of ARA in adipose tissue. It activates its cognate receptor IP-R coupled to the stimulatory $G$ protein (Gs) which in turn increases cAMP levels and activates PKA. When activated by $c P G \mid 2, I P-R$ signaling enhances the differentiation of white adipocyte precursor cells via the expression of $\mathrm{C} / \mathrm{EBP} \beta$ and $\mathrm{C} / \mathrm{EBP} \delta$, both of which being critical for the progression of early phase of adipogenesis and the activation of PPARy [23-25, 33, 34]. In addition to activating a plasma membrane receptor, prostacyclin can also signal through PPARs which in turn bind to specific peroxisome proliferator response elements (PPREs) in the promoter region of target genes to regulate their expression [35]. It is favored by the colocalization of COX-2 and the prostacyclin synthase PGIS at the nuclear membrane [36]. PPARs activation by prostacyclin analogues, such as cPGl2, has been also involved in adipocyte differentiation process [37]. Moreover, it was demonstrated in vitro that treatment with cPGI2 of pre-adipocytes isolated from WAT led to increased expression of brite marker genes, in particular upon $\beta$-adrenergic stimulation [22, 38, 39].

Herein, we demonstrate that both IP-R and PPARs pathways can be activated in human white adipocytes by CPGI2 and cooperate to induce their conversion into brite adipocytes. This is shown by the acquisition of a brite phenotype at the molecular and functional levels. Interestingly, we demonstrate that this conversion is not due to the activation of a single pathway. First, $\mathrm{cPGI} 2$ able to bind to the cell surface receptor IP-R activates the CAMP/PKA/CREB pathway. CREB phosphorylation and its binding to cAMP response element ( $C R E)$ is known to trigger the transactivation of various promoters of adipogenic genes including UCP1 and DIO2 [1]. Second, we determine that $\mathrm{cPGI} 2$ is able to induce brite adipocyte phenotype through activation of PPARY pathway. Assays combining IP-R and PPARy antagonists demonstrate that these two pathways act independently in an additive manner. Both pathways trigger the expression of UCP1 by additive effects of CRE and PPRE-dependent transcriptional activities.

In addition, we show that PPARa allowed only a partial acquisition of cPGI2induced brite adipocyte phenotype. It is known that PPARa signaling is implicated in the augmentation of the lipid oxidative capacity of brite adipocytes [40,41]. One of the key 
proteins of this system is the mitochondrial carnitine acyl transferase CPT1M. It has also been demonstrated that the expression of CPT1M gene is regulated at the transcriptional level by PPARa in muscle cells [42] owing to the presence of a PPRE in its promoter. In hMADS adipocytes, inhibition of PPARa expression and of PPARY activity decrease CPT1M expression in an additive manner. Interestingly, activation of PPARy is able to induce the expression of various genes displaying a PPRE in their promoter (i.e. adiponectin, FABP4, UCP1 and CPT1M), which were not sensitive to PPARa. This discrepancy could be due to the abundance and/or activation of specific co-factors allowing a selective binding to PPREs.

The in vitro capacities of carbaprostacyclin, i.e. recruitment of pre-adipocytes into brite adipogenesis and promotion of the conversion of mature white adipocytes into brite adipocytes, point out this product as a potential therapeutic compound in order to treat obesity and associated pathologies. Prostacyclin and its analogues display vasodilatation property as well as inhibition of platelet aggregation and are already extensively used in human clinic to treat hypertension [43]. Unfortunately, prostacyclin has a very short half-life and is metabolized rapidly into the less potent 6-Keto-PGF1a [43] which represents an important limitation for its use in vivo. To overcome this challenge, many stable analogues of prostacyclin have been synthesized, such as epoprostenol, iloprost and beraprost, with a longer half-life $(\approx 1$ hour $)$ in mice and humans [43-45]. One of these drugs, beraprost sodium (BPS), which promotes a potent vasodilatory effect through activation of IP-R, has already been tested in obesity and diabetes context $[46,47]$. For example, BPS is reported to attenuate inflammation in diabetic patients [46]. Interestingly, chronic treatment with BPS of mice fed a high fat diet decreases plasma and liver triglycerides levels, and improves glucose metabolism and insulin resistance in association with reduction of inflammation of epididymal WAT [47]. Nevertheless, BPS-treated mice and rats do not exhibit a decreased body weight, pointing out the limited effects of IP-R agonists [47, 48]. However, IP-R null mice fed a high fat diet show a resistance to weight gain associated with decreased adipocyte number and increased adipocyte size [23]. Indeed, we and others have demonstrated that the browning potential of cPGI2 was mainly due to the activation of PPARy pathway independently of IP-R activation [20, 22, 38, 39]. This is likely due to a high cell- 
penetrating capacity of cPGI2 compared to other prostacyclin derivatives [49]. The development of a new class of prostacyclin analogs, such as treprostinil, displaying an extended half-life ( 4 hours) and able to activate both IP-R and PPARy, may open a new area to favor browning of white adipose tissue [50].

Herein, we demonstrate that carbaprostacyclin is able to induce the conversion of human white to brite adipocytes through the activation of dual signaling pathways as depicted in Fig. 6. We suggest the development of novel therapies targeting preferentially adipose tissue and based on the use of prostacyclin analogues highly potent to activate IP-R and PPARs pathways, in order to combat obesity and associated diseases via the recruitment and activation of thermogenic adipocytes. 


\section{Figure Legends.}

Figure 1: Effect of cPGI2 treatment on white to brite adipocyte conversion. hMADS cells were differentiated into white (ctrl) or brite (rosi) adipocytes, and treated or not with various doses (A) or with $1 \mu \mathrm{M}$ (B-E) cPGI2 under white adipocyte conditions. (A) UCP1 mRNA expression determined by RT-qPCR. (B) UCP1 and PLNA/B protein levels analyzed by Western blot. $\beta$-tubulin was used as loading control. (C) Oil red $\mathrm{O}$ staining of control and $\mathrm{CPG} 2 \mathrm{2}$ treated adipocytes and corresponding microscopic photomicrographs. (D) Measurement of GPDH activities and (E) mRNA expression of adipogenic markers were analyzed by RT-qPCR. Results are mean $+/-$ SEM of $4(A)$ and $6(E)$ independent experiments. a: $p<0.05$ vs. ctrl.

Figure 2: Molecular and functional effects of cPGI2 on hMADS adipocytes. A and B illustrated the expression of brown/brite adipocyte and mitochondrial markers, respectively. (C) Expression of Zic1 and Tbx1 mRNA as specific marker of brown and brite adipocytes respectively. (D) Cytochrome $c$ oxidase activity measurement. (E, F) Basal, maximal and uncoupled mitochondrial activities, in cPGI2-treated hMADS adipocytes, were evaluated by the determination of oxygen consumption rate (OCR). (G) Mitochondrial DNA content evaluated by qPCR using specific nuclear $(L p l)$ and mitochondrial $(N d 1)$ DNA primers. Plots and histograms display mean \pm SEM, a: $p<0.05$ vs. ctrl, data are from 3 (D) or 6 (A-C, E-G) independent experiments.

Figure 3: Role of IP-R in the effects of $\mathbf{c P G I 2}$. (A) Intracellular $\mathrm{Ca}^{2+}$ concentration analyzed by live fluoromicroscopy using a fluorescent sensitive $\mathrm{Ca}^{++}$probe (Quest Fluo-8). hMADS adipocytes were treated with ionomycin $(60 \mathrm{nM})$ and cPGI2 $(1 \mu \mathrm{M})$ at indicated time. (B) Kinetics of CREB protein phosphorylation, analyzed by Western blot, upon acute treatment of hMADS adipocytes with $1 \mu \mathrm{M} \mathrm{cPGI} 2$ in the absence or in the presence of $5 \mu \mathrm{M}$ CAY10441 as an IP-R antagonist or treated only with $1 \mu \mathrm{M}$ MRE269 as an IP-R agonist. Histograms display ratio of band intensity quantification from 3 experiments for each condition. (C) hMADS adipocytes were treated or not with $1 \mu \mathrm{M}$ MRE269 between days 14 and 17; UCP1, CPT1M and PLN1 mRNA expression were analyzed by RT-qPCR. (D) hMADS adipocytes were treated or not with $5 \mu \mathrm{M}$ CAY10441 between days 14 and 17 in the presence of $1 \mu \mathrm{M}$ CPGI2. UCP1, CPT1M and PLN1 mRNA were analyzed by RT-qPCR. Results are mean \pm SEM of $3(A, B, C)$ and 5 (D) independent experiments. $a: p<0.05$ vs. ctrl or time point 0 min and $b: p<0.05$ vs. cPGl2.

Figure 4: Involvement of PPARy in cPGI2-mediated effects. hMADS cells were treated between days 14 and 17 with $1 \mu \mathrm{M}$ cPGI2 in the absence or the presence of $0.3 \mu \mathrm{M}$ GW9662, a PPARY antagonist or in the presence of $5 \mu \mathrm{M}$ CAY10441, an IP-R antagonist. FABP4, Adiponectin (A), UCP1, CPT1M and PLN1 (B, C and D) mRNA expression was analyzed by RTqPCR. Results are mean +/- SEM of 5 independent experiments. $a: p<0.05$ vs. ctrl and b: $\mathrm{p}<0.05$ vs. cPGI2.

Figure 5: Involvement of PPARa in the CPGI2-induced UCP1 expression. hMADS cells were differentiated into white adipocytes and transfected with $20 \mathrm{nM}$ siRNA ctrl or against PPAR a days 12 and 14 . At day 14, cells were treated or not with $1 \mu \mathrm{M} \mathrm{cPGl}$ in the absence or the 
presence of $0.3 \mu \mathrm{M}$ GW9662, a PPARy antagonist. PPARa, UCP1, PLN1 and CPT1M mRNA expression were analyzed by RT-qPCR. Results are mean of 4 independent experiments. a: $\mathrm{p}<0.05$ vs. ctrl, b: $\mathrm{p}<0.05$ vs. cPGI2 and c: $\mathrm{p}<0.05$ vs. cPGI2+GW9662.

Figure 6: Summary diagram of CPGI2 signaling pathways in hMADS adipocytes. 


\section{References}

[1] B. Cannon, J. Nedergaard, Brown adipose tissue: function and physiological significance, Physiol. Rev., 84 (2004) 277-359.

[2] J. Nedergaard, V. Golozoubova, A. Matthias, A. Asadi, A. Jacobsson, B. Cannon, UCP1: the only protein able to mediate adaptive non-shivering thermogenesis and metabolic inefficiency, Biochim. Biophys. Acta, 1504 (2001) 82-106.

[3] A. Frontini, S. Cinti, Distribution and development of brown adipocytes in the murine and human adipose organ, Cell metabolism, 11 (2010) 253-256.

[4] N. Petrovic, T.B. Walden, I.G. Shabalina, J.A. Timmons, B. Cannon, J. Nedergaard, Chronic peroxisome proliferator-activated receptor gamma (PPARgamma) activation of epididymally derived white adipocyte cultures reveals a population of thermogenically competent, UCP1-containing adipocytes molecularly distinct from classic brown adipocytes, J. Biol. Chem., 285 (2010) 7153-7164.

[5] D. Ricquier, B. Miroux, M. Larose, A.M. Cassard-Doulcier, F. Bouillaud, Endocrine regulation of uncoupling proteins and energy expenditure, Int. J. Obes. Relat. Metab. Disord., 24 Suppl 2 (2000) S86-88.

[6] G. Barbatelli, I. Murano, L. Madsen, Q. Hao, M. Jimenez, K. Kristiansen, J.P. Giacobino, R. De Matteis, S. Cinti, The emergence of cold-induced brown adipocytes in mouse white fat depots is determined predominantly by white to brown adipocyte transdifferentiation, Am. J. Physiol. Endocrinol. Metab., 298 (2010) E1244-1253.

[7] I.G. Shabalina, N. Petrovic, J.M. de Jong, A.V. Kalinovich, B. Cannon, J. Nedergaard, UCP1 in brite/beige adipose tissue mitochondria is functionally thermogenic, Cell reports, 5 (2013) 1196-1203.

[8] Y.H. Lee, E.P. Mottillo, J.G. Granneman, Adipose tissue plasticity from WAT to BAT and in between, Biochim. Biophys. Acta, 1842 (2014) 358-369.

[9] M. Rosenwald, A. Perdikari, T. Rulicke, C. Wolfrum, Bi-directional interconversion of brite and white adipocytes, Nat. Cell Biol., 15 (2013) 659-667.

[10] A.M. Cypess, S. Lehman, G. Williams, I. Tal, D. Rodman, A.B. Goldfine, F.C. Kuo, E.L. Palmer, Y.H. Tseng, A. Doria, G.M. Kolodny, C.R. Kahn, Identification and importance of brown adipose tissue in adult humans, N. Engl. J. Med., 360 (2009) 15091517.

[11] J. Nedergaard, T. Bengtsson, B. Cannon, Unexpected evidence for active brown adipose tissue in adult humans, Am. J. Physiol. Endocrinol. Metab., 293 (2007) E444452.

[12] M. Saito, Y. Okamatsu-Ogura, M. Matsushita, K. Watanabe, T. Yoneshiro, J. NioKobayashi, T. Iwanaga, M. Miyagawa, T. Kameya, K. Nakada, Y. Kawai, M. Tsujisaki, High incidence of metabolically active brown adipose tissue in healthy adult humans: effects of cold exposure and adiposity, Diabetes, 58 (2009) 1526-1531.

[13] W.D. van Marken Lichtenbelt, J.W. Vanhommerig, N.M. Smulders, J.M. Drossaerts, G.J. Kemerink, N.D. Bouvy, P. Schrauwen, G.J. Teule, Cold-activated brown adipose tissue in healthy men, N. Engl. J. Med., 360 (2009) 1500-1508.

[14] K.A. Virtanen, M.E. Lidell, J. Orava, M. Heglind, R. Westergren, T. Niemi, M. Taittonen, J. Laine, N.J. Savisto, S. Enerback, P. Nuutila, Functional brown adipose tissue in healthy adults, N. Engl. J. Med., 360 (2009) 1518-1525. 
[15] A.M. Cypess, A.P. White, C. Vernochet, T.J. Schulz, R. Xue, C.A. Sass, T.L. Huang, C. Roberts-Toler, L.S. Weiner, C. Sze, A.T. Chacko, L.N. Deschamps, L.M. Herder, N. Truchan, A.L. Glasgow, A.R. Holman, A. Gavrila, P.O. Hasselgren, M.A. Mori, M. Molla, Y.H. Tseng, Anatomical localization, gene expression profiling and functional characterization of adult human neck brown fat, Nat. Med., 19 (2013) 635-639.

[16] N.Z. Jespersen, T.J. Larsen, L. Peijs, S. Daugaard, P. Homoe, A. Loft, J. de Jong, N. Mathur, B. Cannon, J. Nedergaard, B.K. Pedersen, K. Moller, C. Scheele, A classical brown adipose tissue mRNA signature partly overlaps with brite in the supraclavicular region of adult humans, Cell metabolism, 17 (2013) 798-805.

[17] L.Z. Sharp, K. Shinoda, H. Ohno, D.W. Scheel, E. Tomoda, L. Ruiz, H. Hu, L. Wang, Z. Pavlova, V. Gilsanz, S. Kajimura, Human BAT possesses molecular signatures that resemble beige/brite cells, PLoS One, 7 (2012) e49452.

[18] J. Wu, P. Bostrom, L.M. Sparks, L. Ye, J.H. Choi, A.H. Giang, M. Khandekar, K.A. Virtanen, P. Nuutila, G. Schaart, K. Huang, H. Tu, W.D. van Marken Lichtenbelt, J. Hoeks, S. Enerback, P. Schrauwen, B.M. Spiegelman, Beige adipocytes are a distinct type of thermogenic fat cell in mouse and human, Cell, 150 (2012) 366-376.

[19] G. Ailhaud, F. Massiera, P. Weill, P. Legrand, J.M. Alessandri, P. Guesnet, Temporal changes in dietary fats: role of $n-6$ polyunsaturated fatty acids in excessive adipose tissue development and relationship to obesity, Prog. Lipid Res., 45 (2006) 203236.

[20] D.F. Pisani, R.A. Ghandour, G.E. Beranger, P. Le Faouder, J.C. Chambard, M. Giroud, A. Vegiopoulos, M. Djedaini, J. Bertrand-Michel, M. Tauc, S. Herzig, D. Langin, G. Ailhaud, C. Duranton, E.Z. Amri, The omega6-fatty acid, arachidonic acid, regulates the conversion of white to brite adipocyte through a prostaglandin/calcium mediated pathway, Molecular metabolism, 3 (2014) 834-847.

[21] L. Madsen, L.M. Pedersen, H.H. Lillefosse, E. Fjaere, I. Bronstad, Q. Hao, R.K. Petersen, P. Hallenborg, T. Ma, R. De Matteis, P. Araujo, J. Mercader, M.L. Bonet, J.B. Hansen, B. Cannon, J. Nedergaard, J. Wang, S. Cinti, P. Voshol, S.O. Doskeland, K. Kristiansen, UCP1 induction during recruitment of brown adipocytes in white adipose tissue is dependent on cyclooxygenase activity, PLoS One, 5 (2010) e11391.

[22] A. Vegiopoulos, K. Muller-Decker, D. Strzoda, I. Schmitt, E. Chichelnitskiy, A. Ostertag, M. Berriel Diaz, J. Rozman, M. Hrabe de Angelis, R.M. Nusing, C.W. Meyer, W. Wahli, M. Klingenspor, S. Herzig, Cyclooxygenase-2 controls energy homeostasis in mice by de novo recruitment of brown adipocytes, Science, 328 (2010) 1158-1161.

[23] F. Massiera, P. Saint-Marc, J. Seydoux, T. Murata, T. Kobayashi, S. Narumiya, P. Guesnet, E.Z. Amri, R. Negrel, G. Ailhaud, Arachidonic acid and prostacyclin signaling promote adipose tissue development: a human health concern?, J. Lipid Res., 44 (2003) 271-279.

[24] J. Aubert, P. Saint-Marc, N. Belmonte, C. Dani, R. Negrel, G. Ailhaud, Prostacyclin IP receptor up-regulates the early expression of C/EBPbeta and C/EBPdelta in preadipose cells, Mol. Cell. Endocrinol., 160 (2000) 149-156.

[25] G. Vassaux, D. Gaillard, G. Ailhaud, R. Negrel, Prostacyclin is a specific effector of adipose cell differentiation. Its dual role as a cAMP- and $\mathrm{Ca}(2+)$-elevating agent, J. Biol. Chem., 267 (1992) 11092-11097.

[26] B. Jia, L. Madsen, R.K. Petersen, N. Techer, R. Kopperud, T. Ma, S.O. Doskeland, G. Ailhaud, J. Wang, E.Z. Amri, K. Kristiansen, Activation of protein kinase a and 
exchange protein directly activated by cAMP promotes adipocyte differentiation of human mesenchymal stem cells, PLoS One, 7 (2012) e34114.

[27] A.K. Hihi, L. Michalik, W. Wahli, PPARs: transcriptional effectors of fatty acids and their derivatives, Cell Mol Life Sci, 59 (2002) 790-798.

[28] C. Elabd, C. Chiellini, M. Carmona, J. Galitzky, O. Cochet, R. Petersen, L. Penicaud, K. Kristiansen, A. Bouloumie, L. Casteilla, C. Dani, G. Ailhaud, E.Z. Amri, Human multipotent adipose-derived stem cells differentiate into functional brown adipocytes, Stem Cells, 27 (2009) 2753-2760.

[29] D.F. Pisani, M. Djedaini, G.E. Beranger, C. Elabd, M. Scheideler, G. Ailhaud, E.Z. Amri, Differentiation of Human Adipose-Derived Stem Cells into "Brite" (Brown-in-White) Adipocytes, Front. Endocrinol. (Lausanne), 2 (2011) 87.

[30] A.M. Rodriguez, C. Elabd, F. Delteil, J. Astier, C. Vernochet, P. Saint-Marc, J. Guesnet, A. Guezennec, E.Z. Amri, C. Dani, G. Ailhaud, Adipocyte differentiation of multipotent cells established from human adipose tissue, Biochem. Biophys. Res. Commun., 315 (2004) 255-263.

[31] R. Negrel, P. Grimaldi, G. Ailhaud, Establishment of preadipocyte clonal line from epididymal fat pad of ob/ob mouse that responds to insulin and to lipolytic hormones, Proc. Natl. Acad. Sci. U. S. A., 75 (1978) 6054-6058.

[32] M.D. Brand, D.G. Nicholls, Assessing mitochondrial dysfunction in cells, Biochem. J., 435 (2011) 297-312.

[33] G. Vassaux, D. Gaillard, C. Darimont, G. Ailhaud, R. Negrel, Differential response of preadipocytes and adipocytes to prostacyclin and prostaglandin E2: physiological implications, Endocrinology, 131 (1992) 2393-2398.

[34] J. Aubert, G. Ailhaud, R. Negrel, Evidence for a novel regulatory pathway activated by (carba)prostacyclin in preadipose and adipose cells, FEBS Lett., 397 (1996) 117-121.

[35] B.M. Forman, J. Chen, R.M. Evans, Hypolipidemic drugs, polyunsaturated fatty acids, and eicosanoids are ligands for peroxisome proliferator-activated receptors alpha and delta, Proc. Natl. Acad. Sci. U. S. A., 94 (1997) 4312-4317.

[36] H. Lim, S.K. Dey, A novel pathway of prostacyclin signaling-hanging out with nuclear receptors, Endocrinology, 143 (2002) 3207-3210.

[37] R.P. Brun, P. Tontonoz, B.M. Forman, R. Ellis, J. Chen, R.M. Evans, B.M. Spiegelman, Differential activation of adipogenesis by multiple PPAR isoforms, Genes Dev, 10 (1996) 974-984.

[38] I. Bayindir, R. Babaeikelishomi, S. Kocanova, I.S. Sousa, S. Lerch, O. Hardt, S. Wild, A. Bosio, K. Bystricky, S. Herzig, A. Vegiopoulos, Transcriptional Pathways in cPGl2-Induced Adipocyte Progenitor Activation for Browning, Front. Endocrinol. (Lausanne), 6 (2015) 129.

[39] K. Mossenbock, A. Vegiopoulos, A.J. Rose, T.P. Sijmonsma, S. Herzig, T. Schafmeier, Browning of white adipose tissue uncouples glucose uptake from insulin signaling, PLoS One, 9 (2014) e110428.

[40] C. Ribet, E. Montastier, C. Valle, V. Bezaire, A. Mazzucotelli, A. Mairal, N. Viguerie, D. Langin, Peroxisome proliferator-activated receptor-alpha control of lipid and glucose metabolism in human white adipocytes, Endocrinology, 151 (2010) 123-133.

[41] B. Xue, A. Coulter, J.S. Rim, R.A. Koza, L.P. Kozak, Transcriptional synergy and the regulation of Ucp1 during brown adipocyte induction in white fat depots, Mol. Cell. Biol., 25 (2005) 8311-8322. 
[42] A. Baldan, J. Relat, P.F. Marrero, D. Haro, Functional interaction between peroxisome proliferator-activated receptors-alpha and Mef-2C on human carnitine palmitoyltransferase 1 beta (CPT1beta) gene activation, Nucleic Acids Res, 32 (2004) 4742-4749.

[43] H. Olschewski, F. Rose, R. Schermuly, H.A. Ghofrani, B. Enke, A. Olschewski, W. Seeger, Prostacyclin and its analogues in the treatment of pulmonary hypertension, Pharmacol Ther, 102 (2004) 139-153.

[44] M. Hildebrand, Pharmacokinetics of iloprost and cicaprost in mice, Prostaglandins, 44 (1992) 431-442.

[45] M. Hildebrand, T. Staks, B. Nieuweboer, Pharmacokinetics and pharmacodynamics of cicaprost in healthy volunteers after oral administration of 5 to 20 micrograms, Eur $\mathrm{J}$ Clin Pharmacol, 39 (1990) 149-153.

[46] K. Fujiwara, A. Nagasaka, M. Nagata, K. Yamamoto, S. Imamura, N. Oda, Y. Sawai, N. Hayakawa, A. Suzuki, M. Itoh, A stable prostacyclin analogue reduces high serum TNF-alpha levels in diabetic patients, Exp Clin Endocrinol Diabetes, 112 (2004) 390-394.

[47] E. Inoue, T. Ichiki, K. Takeda, H. Matsuura, T. Hashimoto, J. Ikeda, A. Kamiharaguchi, K. Sunagawa, Beraprost sodium, a stable prostacyclin analogue, improves insulin resistance in high-fat diet-induced obese mice, J Endocrinol, 213 (2012) 285-291.

[48] N. Sato, M. Kaneko, M. Tamura, H. Kurumatani, The prostacyclin analog beraprost sodium ameliorates characteristics of metabolic syndrome in obese Zucker (fatty) rats, Diabetes, 59 (2010) 1092-1100.

[49] T. Hatae, M. Wada, C. Yokoyama, M. Shimonishi, T. Tanabe, Prostacyclindependent apoptosis mediated by PPAR delta, J. Biol. Chem., 276 (2001) 46260-46267. [50] K. Laliberte, C. Arneson, R. Jeffs, T. Hunt, M. Wade, Pharmacokinetics and steadystate bioequivalence of treprostinil sodium (Remodulin) administered by the intravenous and subcutaneous route to normal volunteers, J Cardiovasc Pharmacol, 44 (2004) 209214. 


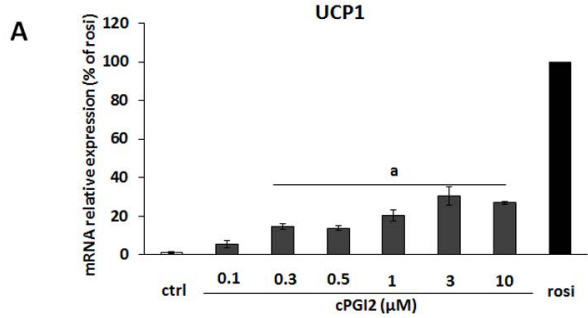

B

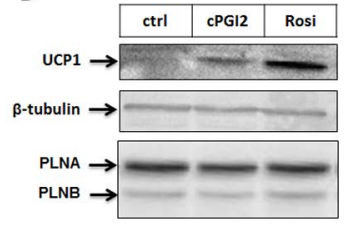

D

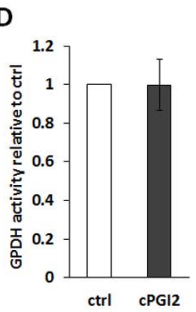

E

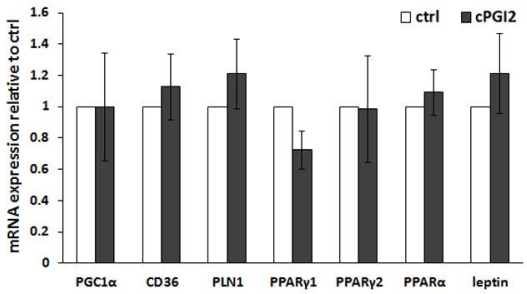


A

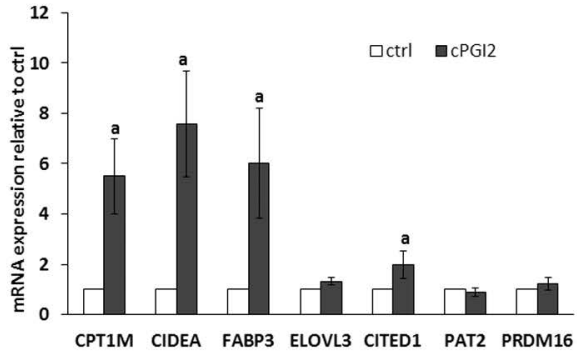

B

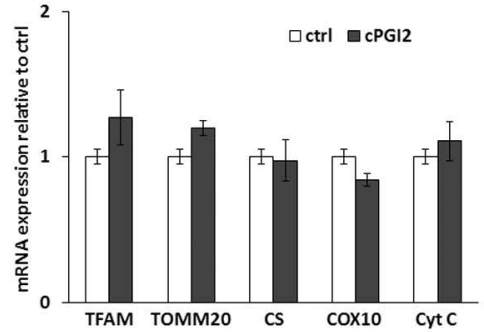

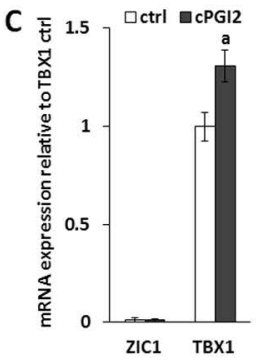

F

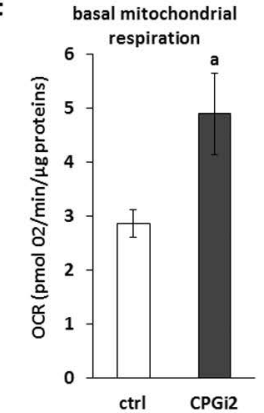

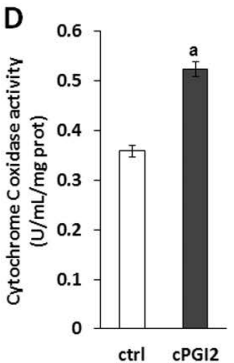

E
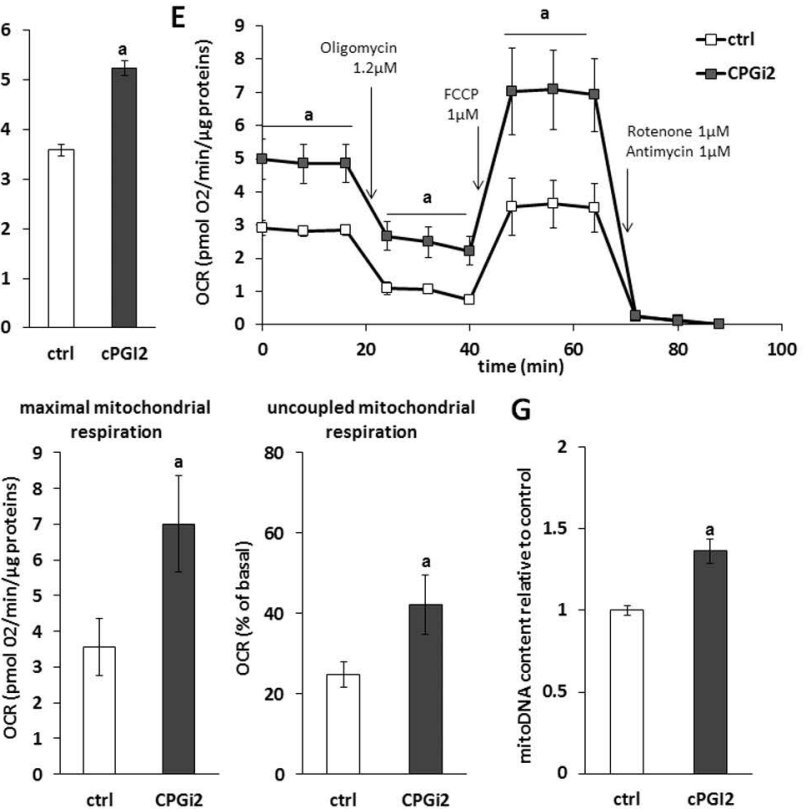
A

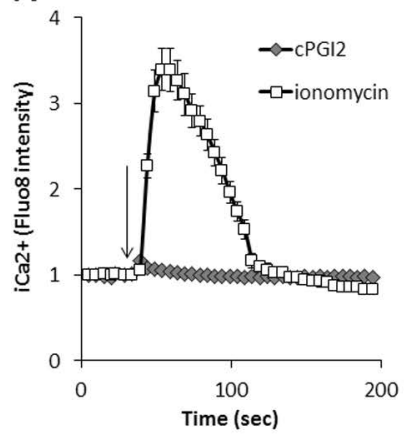

C

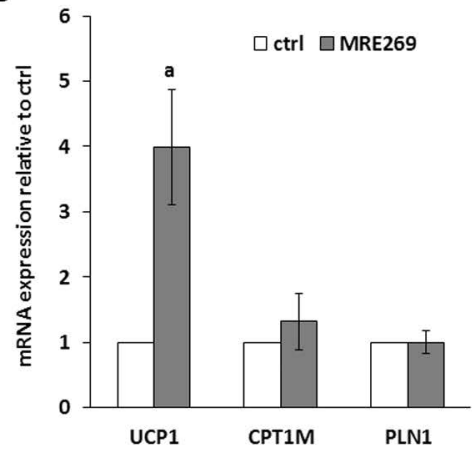

B

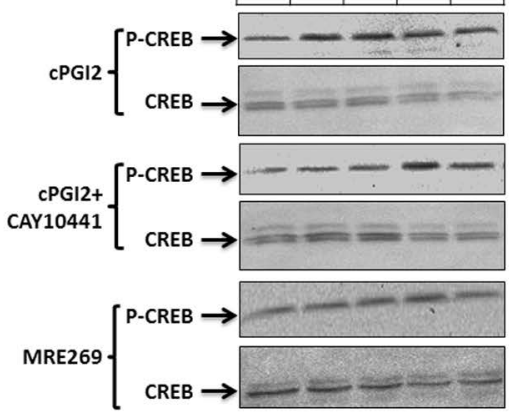

P-CREB/CREB (a.u.)
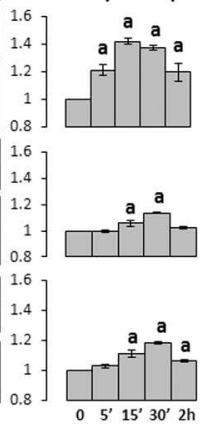

D

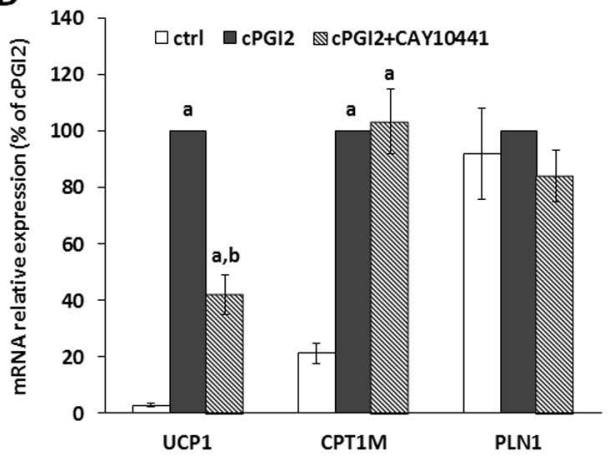




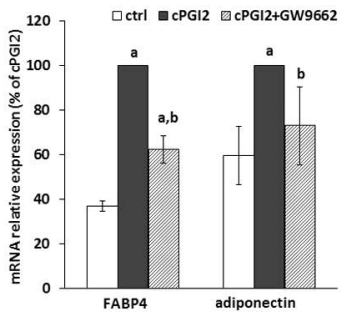

C

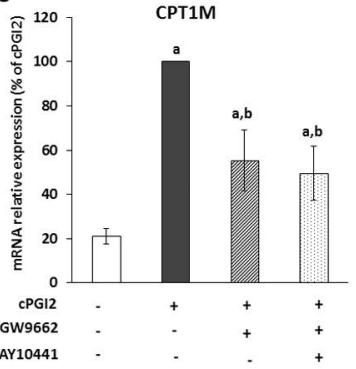

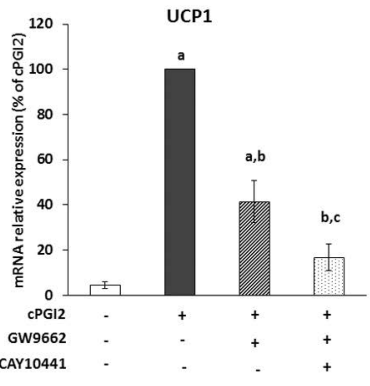

D

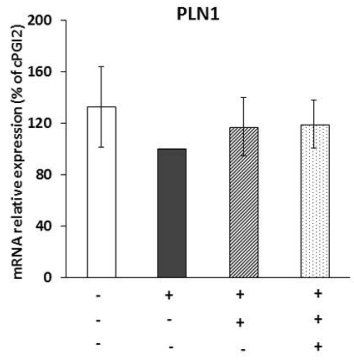


A

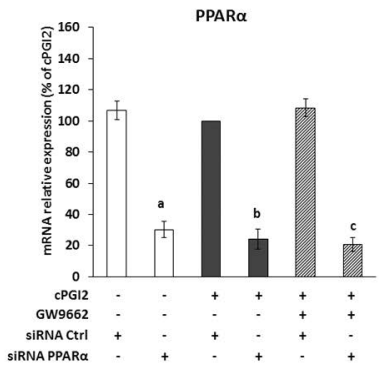

C

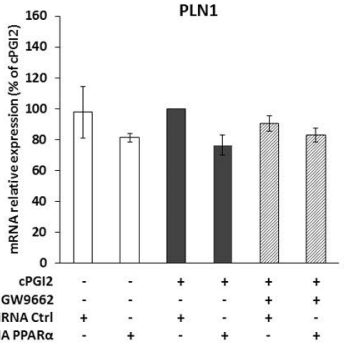

B

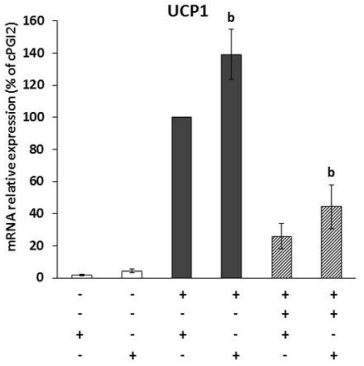

D

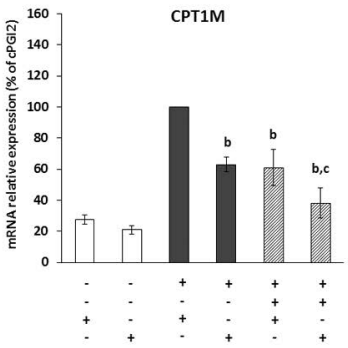




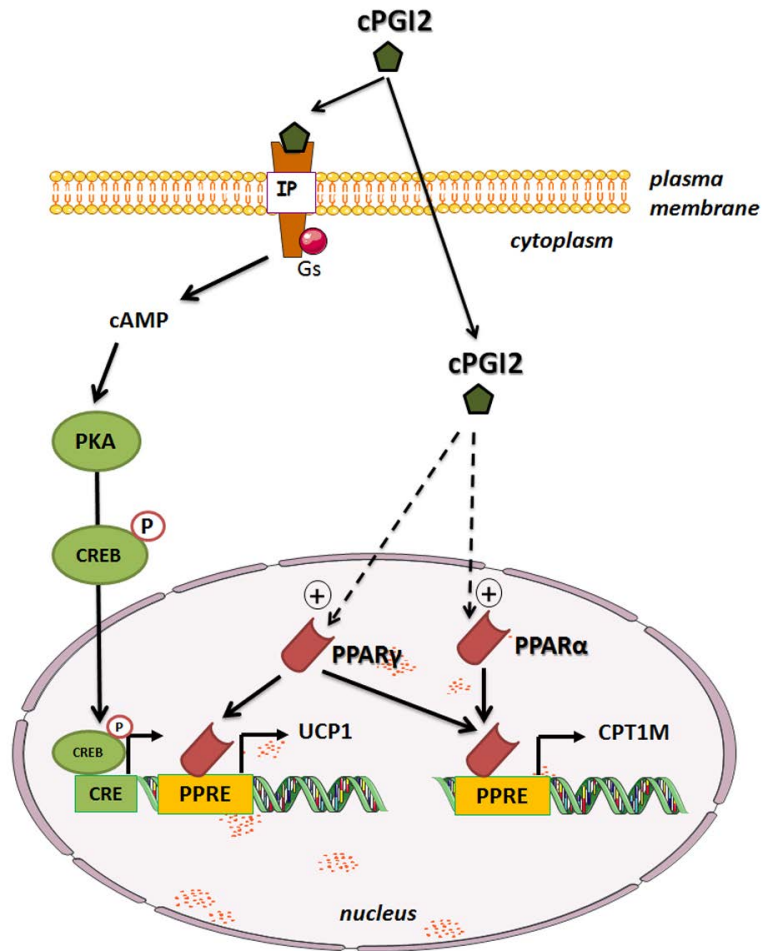

